\title{
Les recommandations de I'ASSM «Structures de soutien éthique en médecine» en consultation
}

\section{Samia Hurst ${ }^{a}$, \\ Michelle Salathéb}

a Prof., Institut d'éthique biomédicale, Université de Genève

b lic. iur., MAE, Secrétaire générale adjointe de l'ASSM

* Les personnes suivantes font partie de la sous-commission: Prof. Samia Hurst, Genève (Présidente); Dr Christof Arn Scharans; Dr Charles

Chappuis, Spiegel; Dr Carlo Foppa, Morges; Irma Graf, Saint-Gall; Prof. Annemarie Kesselring, Berne; Prof. Christian Kind, Saint-Gall, Président CCE; Angelika Lehmann, BNS, MAS Ethique Bâle; Dr Barbara Meyer-Zehnder, Bâle; Dr Rouven Porz, Berne; lic. iur., MAE Michelle Salathé, Bâle, Secrétaire générale adjointe de l'ASSM; Dr Regula Schmitt, Ittigen.

Correspondance: Académie Suisse des Sciences Médicales (ASSM) Petersplatz 13 CH-4051 Bâle

mail[at]samw.ch
Les difficultés éthiques sont fréquentes dans la pratique de la médecine. A titre d'exemple les décisions de continuer ou d'interrompre des mesures de maintien en vie lorsque l'espoir est faible, le traitement de nouveau-nés à la limite de la viabilité, le respect et les limites de l'auto-détermination du patient, la distribution juste des ressources dans un contexte de pressions croissantes sur les coûts de la santé, toutes ces questions soulèvent des conflits de valeurs autour d'enjeux importants. Des recommandations et directives médico-éthiques, comme par exemple celles de l'Académie Suisse des Sciences Médicales (ASSM), offrent des lignes directrices et un soutien aux professionnels de la santé qui sont confrontés à de telles situations. Ces recommandations et directives ne peuvent en revanche offrir que des considérations générales. Dans les cas concrets, la réflexion et la décision devront être adaptées au contexte et aux spécificités de la situation individuelle. Les structures de soutien éthique en médecine, qui peuvent prendre plusieurs formes, offrent un soutien à ces processus.

Dans plusieurs de ses directives, l'ASSM recommande ainsi le recours aux structures de soutien éthique en médecine, qui offrent un complément à la délibération éthique conduite au sein des équipes dans le quotidien clinique. En Suisse, de nombreux concepts ont été développés dans ce domaine ces dernières années, qui vont de l'avis éthique dans un cas individuel jusqu'à l'aide à l'élaboration de directives concernant des problèmes éthiques, en passant par l'organisation de sessions de formation continue en éthique.

Toutefois, les structures d'éthique ne sont utiles que lorsqu'elles remplissent leurs missions de façon adéquate. C'est pourquoi, la Commission Centrale d'Ethique a chargé une sous-commission* d'élaborer des recommandations concernant la qualité et la durabilité de la prise de décision d'éthique clinique. Le projet de texte des recommandations «Structures de soutien éthique en médecine» est maintenant disponible. Elles présentent en introduction les buts et tâches des structures d'éthique tout en attirant l'attention sur les dangers et les malentendus qui y sont liés.

En première partie, les recommandations soulignent la diversité des structures et méthodes de soutien éthique et décrivent les éléments clés de leur travail: A quel moment le recours à une structure d'éthique est-il pertinent? Quelle attitude fondamen- tale les membres d'une structure d'éthique doiventils adopter? Quelles connaissances et compétences doivent-ils apporter? Quels aspects doivent être considérés dans la prise de décisions individuelles, dans l'élaboration de recommandations éthiques générales ou dans la formation prégraduée, postgraduée et continue? La deuxième partie aborde des conseils pour l'établissement de structures d'éthique. Quelles sont les conditions structurelles requises pour l'établissement d'une structure d'éthique? Comment l'indépendance d'une structure d'éthique peut-elle être garantie? Quelle devrait être sa composition? Et quels sont les éléments susceptibles d'améliorer sa visibilité?

Enfin le chapitre 3.5. donne des indications pratiques, sous forme de checkliste, pour le choix de la structure adéquate dans des cas individuels et pour l'organisation du processus de consultation.

Les structures d'éthique se sont développées surtout dans les grands hôpitaux de soins aigus, mais s'établissent de plus en plus dans d'autres domaines comme la psychiatrie, la pédiatrie ou les soins de longue durée, tant dans le secteur ambulatoire que dans le secteur stationnaire. Dans l'annexe aux recommandations, les exigences requises pour les structures d'éthique dans les différents domaines sont récapitulées, différentes méthodes de soutien éthique sont présentées et des réflexions sur la qualité sont formulées.

Ces recommandations s'adressent aux éthiciens cliniques, aux membres de comités d'éthique clinique, et à toutes autres personnes explicitement engagées dans l'offre de consultations, conseils, et autres avis concernant les enjeux éthiques rencontrés dans la pratique clinique. Elles s'adressent également aux institutions hospitalières et ambulatoires (indépendamment de leur taille) qui souhaiteraient mettre sur pied ou qui viennent d'établir un soutien éthique, ainsi qu'aux professionnels de la santé qui font appel à un soutien éthique dans la pratique clinique quotidienne, ou qui en ressentent le besoin.

Lors de sa séance du 24 novembre 2011, le Sénat a adopté ces recommandations pour la procédure de consultation qui durera jusqu'à fin février 2012; l'avis de toutes les institutions et personnes intéressées est sollicité.

Les recommandations peuvent être consultées sur le site internet de l'ASSM: (www.assm.ch/fr/Ethique/ Directives/en-consultation.html 